\title{
THE BIOMETRICAL GENETICS OF AUTOTETRAPLOIDS I. GENERATIONS DERIVED FROM A CROSS BETWEEN TWO PURE LINES
}

\author{
R. J. KILLICK \\ Scottish Plant Breeding Station, Pentlandfield, Roslin, Midlothion
}

Received 10.xi.70

\section{INTRODUCTION}

BIOMETRICAL genetics, the study of quantitative inheritance, has been almost exclusively concerned with diploid species and at this level of ploidy has had outstanding success as a fundamental science and proved a useful adjunct to plant and animal breeding programmes. There are, however, several crop plants (e.g. potatoes, coffee, lucerne and several forage grasses) which are autotetraploids and in which the pattern of inheritance is tetrasomic, not disomic. The considerable breeding effort which has gone into the improvement of these cultivars has therefore had to proceed without the benefit of reliable information about the genetical architecture of the metrical traits under selection. A knowledge of the types of gene action underlying these traits should help the efficiency of selection by indicating the most appropriate choice of parents and the most suitable method of selection by which to proceed.

In this paper consideration will be given to the information that can be obtained from the generations derived from a cross between two autotetraploid homozygous lines. It therefore follows directly on from the work of Mather and Jinks (1971).

\section{Notation}

With two alleles, $A$ and $a$, at a locus (the maximum when starting with two homozygous lines) there are three possible genotypes in diploids ( $A A$, $A a, a a)$ but five in tetraploids. These are

$\begin{array}{lll}A A A A \text { or } A_{4} & \text { quadruplex } \\ A A A a & \text { or } A_{3} a & \text { triplex } \\ A A a a & \text { or } A_{2} a_{2} & \text { duplex } \\ A a a a & \text { or } A a_{3} & \text { simplex } \\ \text { aaaa } & \text { or } a_{4} & \text { nulliplex }\end{array}$

These must be ascribed algebraic values before the expectations of any statistics can be calculated. By analogy to diploids (Mather, 1949) we might have

$$
\begin{aligned}
A_{4} & =m+d \\
A_{3} a & =A_{2} a_{2}=A a_{3}=m+h \\
a_{4} & =m-d
\end{aligned}
$$

where $m$ is the midparentvalue, but this denies any possibility that the triplex, duplex and simplex genotypes have different values, as they would if, for 
instance, the genetic effect was proportional to the dosage of one allele. This can be overcome by altering the triplex and simplex values to

$$
\begin{aligned}
& A_{3} a=m+\frac{1}{2} d+\frac{1}{2} h \\
& A a_{3}=m-\frac{1}{2} d+\frac{1}{2} h
\end{aligned}
$$

which expresses their intermediate state between the duplex and homozygous genotypes. With complete dominance, however, the value of the simplex genotype becomes $m$ when it would be expected to become $m+d(=m+h)$, so this scheme has a serious shortcoming. Dessureaux (1959) attempted to provide a solution by giving the triplex and simplex genotypes values of

where

$$
\begin{aligned}
& A_{3} a=m+\lambda d \\
& A a_{3}=m-\lambda d
\end{aligned}
$$

or

$$
\begin{aligned}
& \lambda=\frac{1}{2}-\frac{1}{2} h / d \text { if } h \leqslant d \\
& \lambda=\frac{1}{2}+\frac{1}{2} h / d \text { if } h \leqslant-d .
\end{aligned}
$$

He showed the effect of this was to give the triplex and simplex genotypes values of

$$
\begin{aligned}
& A_{3} a=m+\frac{1}{2} d+\frac{1}{2} h \\
& A a_{3}=m-\frac{1}{2} d+\frac{3}{2} h .
\end{aligned}
$$

The difficulty here is that the parameters are not independent but are defined in terms of each other.

Secondly, the simplex genotype makes a contribution to the $h$ parameter three times that of the triplex genotype which is bound to create ambiguity in the interpretation of the dominance parameter, especially from the second-degree statistics where the effect is squared.

On the whole the safest general scheme appears to be to allow the triplex, duplex and simplex genotypes to take unique values of $h_{3}, h_{2}$ and $h_{1}$ respectively (Mather and Jinks, 1971). Whilst this increases the number of parameters it avoids making any assumptions about the relative genetical values of the genotypes.

During polyploid meiosis sister chromatids may enter the same gamete. This process, known as double reduction (Mather, 1936), results from recombination between the centromere and distal genes followed by a particular orientation of the chromosomes on the second metaphase spindle. The coefficient of double reduction $(\alpha)$ represents the proportion of gametes in which sister genes occur. $\alpha$ has a theoretical maximum limit of $\frac{1}{7}(0 \cdot 1428)$ and a minimum value of zero (when segregation is "chromosomal ", i.e. the genes are transmitted as if they were completely linked to the centromere). The five genotypes produce gametes in the proportions shown in table 1 (Fisher, 1949).

TABLE 1

Gametic output in an autotetraploid

Parental genotype

$A_{4}$

$A_{3} a$

$A_{2} a_{2}$

$\mathrm{Aa}_{3}$

$a_{4}$

Gametes

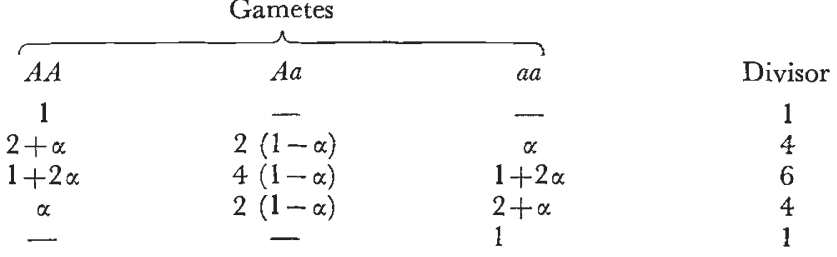


Table 2

Expected genotypic proportions of generation means with double reduction

Quadruplex, $d$

\section{Generation}

$P_{1}$

$P_{2}$

$\mathrm{F}_{1}$

$\mathrm{F}_{2}$

$\mathrm{F}_{3}$

$\mathrm{F}_{4}$

$\mathrm{F}_{2}$ bip

$F_{2} \times P_{1}$

$\mathrm{F}_{2} \times \mathrm{P}_{2}$

$\mathrm{F}_{2} \times \mathrm{F}_{1}$

$\mathrm{B}_{1} \times \mathrm{F}_{1}$

$\mathrm{B}_{2} \times \mathrm{F}_{1}$

$\mathrm{B}_{1}$

$\mathrm{B}_{2}$

$\mathrm{B}_{11}$

$\mathrm{B}_{12}$

$\mathrm{B}_{22}$

$\mathrm{B}_{21}$

$\mathrm{B}_{1}$ self

$\mathrm{B}_{2}$ self

$B_{1}$ bip

$\mathrm{B}_{2}$ bip

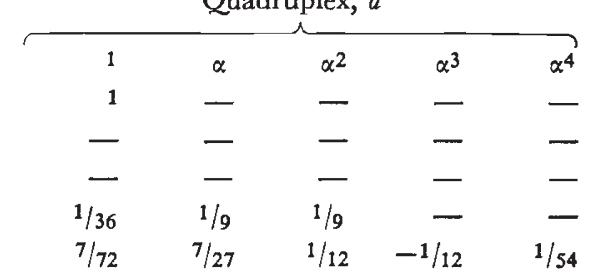

$4 / 81 \quad 14 / 81$

$2 / 9$

- $1 / 27$

$19 / 216$

$1 / 216$

$1 / 6$

$19 / 36$

$-$

$1 / 36$

$73 / 216$

$1 / 216$

$361 / 1296$

$1 / 1296$
$14 / 81 \quad 11 / 108 \quad-7 / 81 \quad 1 / 81$

$7 / 18 \quad-1 / 9$

$2 / 9 \quad 1 / 12 \quad-1 / 54$

$1 / 18 \quad 1 / 12 \quad-1 / 54$

$1 / 3$

$\begin{array}{rrr}- & - & - \\ 5 / 18 & -1 / 18 & -\end{array}$

- $-\quad-\quad-$

$\begin{array}{rrrr}- & - & - & - \\ 5 / 18 & -1 / 18 & - & -\end{array}$

$13 / 36 \quad-5 / 72 \quad-1 / 216 \quad-$

$1 / 36$

$7 / 72 \quad-1 / 216$

$1 / 54-10 / 324 \quad 1 / 324$

$2 / 27-10 / 324 \quad 1 / 324$ $\begin{array}{lll}5 / 36 & - & - \\ 1 / 9 & -1 / 27\end{array}$
Triplex, $h_{3}$

$\begin{array}{rrrrr}1 & \alpha & \alpha^{2} & \alpha^{3} & \alpha^{4} \\ - & - & - & - & - \\ - & - & - & - & - \\ - & - & - & - & - \\ 2 / 9 & 2 / 9 & -4 / 9 & - & - \\ 2 / 9 & 2 / 27 & -5 / 9 & 1 / 3 & -2 / 27\end{array}$

$\begin{array}{rrrrr}20 / 81 & 7 / 81 & -17 / 27 & 28 / 81 & -4 / 81 \\ 5 / 9 & -7 / 9 & 2 / 9 & - & - \\ - & - & - & - & - \\ 13 / 54 & 1 / 6 & -5 / 9 & 4 / 27 & - \\ 23 / 54 & -1 / 9 & -7 / 18 & 2 / 27 & - \\ 5 / 54 & 2 / 9 & -7 / 18 & 2 / 27 & - \\ 2 / 3 & -2 / 3 & - & - & - \\ - & - & - & - & - \\ 4 / 9 & -5 / 9 & 1 / 9 & - & - \\ - & - & - & - & - \\ - & - & - & - & - \\ 4 / 9 & -5 / 9 & 1 / 9 & - & - \\ 10 / 27 & -7 / 18 & - & 1 / 54 & - \\ 1 / 27 & 5 / 18 & -1 / 3 & 1 / 54 & - \\ 38 / 81 & -55 / 162 & -13 / 54 & 10 / 81 & -1 / 81 \\ 2 / 81 & 35 / 162 & -57 / 162 & 10 / 81 & -1 / 81\end{array}$

Nulliplex, $-d$

\begin{tabular}{|c|c|c|c|c|c|c|c|c|c|c|c|c|c|c|}
\hline \multicolumn{4}{|c|}{ Duplex, $h_{2}$} & \multicolumn{6}{|c|}{ Simplex, $h_{1}$} & \multicolumn{5}{|c|}{ Nulliplex, $-d$} \\
\hline 1 & $\alpha$ & $\alpha^{2}$ & $\alpha^{3}$ & $\alpha^{4}$ & 1 & $\alpha$ & $\alpha^{2}$ & $\alpha^{3}$ & $\alpha^{4}$ & 1 & $\alpha$ & $\alpha^{2}$ & $\alpha^{3}$ & $\alpha^{4}$ \\
\hline- & - & - & - & - & - & - & - & - & - & - & - & - & - & - \\
\hline - & - & - & - & - & - & - & - & - & - & 1 & - & - & - & - \\
\hline 1 & - & - & - & - & - & - & - & - & - & - & - & - & - & - \\
\hline $1 / 2$ & $-2 / 3$ & $2 / 3$ & - & - & $2 / 9$ & $2 / 9$ & $-4 / 9$ & - & - & $1 / 36$ & $1 / 9$ & $1 / 9$ & - & - \\
\hline $\begin{array}{l}13 / 36 \\
\text { Tex }\end{array}$ & $-2 / 3$ & $51 / 54$ & $-1 / 2$ & $1 / 9$ & $2 / 9$ & $2 / 27$ & $-5 / 9$ & $1 / 3$ & $-2 / 27$ & $7 / 72$ & $7 / 27$ & $1 / 12$ & $-1 / 12$ & $1 / 54$ \\
\hline $11 / 27$ & $-14 / 27$ & $19 / 18$ & $-14 / 27$ & $2 / 272$ & $20 / 81$ & $7 / 81$ & $-17 / 27$ & $28 / 81$ & $-4 / 81$ & $4 / 81$ & $14 / 81$ & $11 / 108$ & $-7 / 81$ & $1 / 81$ \\
\hline $2 / 9$ & $7 / 18$ & $-1 / 9$ & - & - & - & - & - & - & - & - & - & - & - & - \\
\hline $2 / 9$ & $7 / 18$ & $-1 / 9$ & - & - & $5 / 9$ & $-7 / 9$ & $2 / 9$ & - & - & $2 / 9$ & $7 / 18$ & $-1 / 9$ & - & - \\
\hline $4 / 9$ & $-11 / 18$ & $8 / 9$ & $-2 / 9$ & -1 & $13 / 54$ & $1 / 6$ & $-5 / 9$ & $4 / 27$ & - & $1 / 27$ & $5 / 36$ & $1 / 9$ & $-1 / 27$ & - \\
\hline $7 / 18$ & $-7 / 18$ & $11 / 18$ & $-1 / 9$ & - & $5 / 54$ & $2 / 9$ & $-7 / 18$ & $2 / 27$ & - & $1 / 216$ & $1 / 18$ & $1 / 12$ & $-1 / 54$ & - \\
\hline $7 / 18$ & $-7 / 18$ & $11 / 18$ & $-1 / 9$ & -2 & $23 / 54$ & $-1 / 9$ & $-7 / 18$ & $2 / 27$ & - & $19 / 216$ & $2 / 9$ & $1 / 12$ & $-1 / 54$ & - \\
\hline $1 / 6$ & $1 / 3$ & - & - & - & - & - & - & - & - & - & - & - & - & - \\
\hline $1 / 6$ & $1 / 3$ & - & - & - & $2 / 3$ & $-2 / 3$ & - & - & - & $1 / 6$ & $1 / 3$ & - & - & - \\
\hline $1 / 36$ & $5 / 18$ & $-1 / 18$ & - & - & - & - & - & - & - & - & - & 一 & - & - \\
\hline $19 / 36$ & $5 / 18$ & $-1 / 18$ & - & - & $4 / 9$ & $-5 / 9$ & $1 / 9$ & - & - & $1 / 36$ & $5 / 18$ & $-1 / 18$ & - & - \\
\hline $1 / 36$ & $5 / 18$ & $-1 / 18$ & - & - & $4 / 9$ & $-5 / 9$ & $1 / 9$ & - & - & $19 / 36$ & $5 / 18$ & $-1 / 18$ & - & - \\
\hline $19 / 36$ & $5 / 18$ & $-1 / 18$ & - & - & - & - & - & - & - & - & - & - & - & - \\
\hline $1 / 4$ & $-5 / 18$ & $11 / 36$ & $-1 / 36$ & - & $1 / 27$ & $5 / 18$ & $-1 / 3$ & $1 / 54$ & - & $1 / 216$ & $1 / 36$ & $7 / 72$ & $-1 / 216$ & - \\
\hline $1 / 4$ & $-5 / 18$ & $11 / 36$ & $-1 / 36$ & -1 & $10 / 27$ & $-7 / 18$ & - & $1 / 54$ & - & $73 / 216$ & $13 / 36$ & $-5 / 72$ & $-1 / 216$ & - \\
\hline & $-10 / 54$ & $1 / 2$ & $-10 / 54$ & $1 / 54$ & $2 / 81$ & $35 / 162$ & $-57 / 162$ & $10 / 81$ & & $1 / 1296$ & $5 / 324$ & $2 / 27$ & $-10 / 324$ & $1 / 324$ \\
\hline $7 / 648$ & $-10 / 54$ & $1 / 2$ & $-10 / 54$ & $1 / 543$ & $38 / 81$ & $55 / 162$ & $-13 / 54$ & $10 / 81$ & $-1 / 81$ & $361 / 1296$ & $95 / 324$ & $1 / 54$ & $-10 / 324$ & $1 / 324$ \\
\hline
\end{tabular}




\section{First-DEGREe STATISTICS}

(a) Generation means

The expected means of 22 generations which can be derived from an initial cross between two inbred lines are shown in table 2 in the form of proportions of the five genotypes. The algebraic mean is easily derived by adding $m$, the midparent value $\left(=\frac{1}{2}\left(\mathbf{P}_{1}+\mathbf{P}_{2}\right)\right)$ and putting $d=\frac{1}{2}$ (quadruplex - nulliplex) and $h_{3}, h_{2}$ and $h_{1}$ equal to the triplex, duplex and simplex genotypes respectively. The generations are the two parents, the selfing series from $F_{1}-F_{4}$, biparental progenies $\left(F_{2}\right.$ bip), the first and second backcrosses, the first back-crosses selfed, sibbed ( $B_{1}$ bip, $B_{2}$ bip) and crossed to the $F_{1}$, and the $F_{2}$ crossed to the parents and $F_{1}$. The complexity caused by double reduction is well illustrated by the selfing series. The $F_{4}$ mean not shown in table 2 is

$$
\begin{aligned}
\overline{\mathrm{F}}_{4}= & A_{4}, a_{4} ; \frac{1}{3888}\left(633+1382 \alpha-64 \alpha^{2}-438 \alpha^{3}\right. \\
& \left.+258 \alpha^{4}-78 \alpha^{5}+12 \alpha^{6}\right) \\
& A_{3} a, A a_{3} ; \frac{1}{3888}\left(744-120 \alpha-1896 \alpha^{2}+2040 \alpha^{3}\right. \\
& \left.-1032 \alpha^{4}+312 \alpha^{5}-48 \alpha^{6}\right) \\
& A_{2} a_{2} ; \frac{1}{3888}\left(1134-2520 \alpha+3924 \alpha^{2}-3204 \alpha^{3}\right. \\
& \left.+1548 \alpha^{4}-468 \alpha^{5}+72 \alpha^{6}\right) .
\end{aligned}
$$

Terms to the sixth power of $\alpha$ will, of course, be small even when $\alpha=0 \cdot 14$. Even if segregation is chromosomal the expectations are more complex than experienced in diploids. As in diploids, though, there is a symmetry about the means. For instance in the selfing series, $F_{2}$ bip, and $F_{2} \times F_{1}$ the coefficient of $A_{4}$ is the same as that of $a_{4}$ and the coefficient of $A_{3} a$ is the same as that for $A a_{3}$. In the first and second back-crosses the coefficient of $A_{4}$ is the same as that of $A_{2} a_{2}$, and the coefficient of $A_{3} a$ is the same as that of $A a_{3}$, in the appropriate reciprocal cross. Similar correspondences between reciprocal crosses of the same generation can be seen in table 2 .

Unlike diploids, the $\mathrm{F}_{2}$ mean does not equal the $\mathrm{F}_{2}$ bip mean, nor in the back-cross generations is the coefficient of the additive parameter equal to that of the dominance parameter, though for $B_{1}$ the coefficient of the duplex genotype equals that of the quadruplex (or the nulliplex for $B_{2}$ ). Table 3 shows the proportions of the five genotypes for each generation for $\alpha=0.0$ and $\alpha=0 \cdot 14$. It is clear from this that the decline in heterozygosity on selfing is considerably slower than in diploids.

\section{(b) Effect of double reduction}

Whilst table 3 indicates the extreme effects of double reduction it does not show the effect of double reduction at intermediate values of $\alpha$. This is shown in figs. 1-3. Only one reciprocal of each generation is shown; it is a straightforward matter to infer the changes occurring in the other reciprocal using table 2. For instance, fig. 2 shows only $B_{11}$ of the four second back-crosses, but using table 2 we see that the coefficient of the quadruplex for $B_{11}$ equals that of the duplex for $B_{12}$ and $B_{21}$, and that of the nulliplex for $B_{22}$; the coefficient of the triplex for $B_{11}$ equals that of the triplex for $B_{21}$ and of the simplex for $B_{12}$ and $B_{22}$; and the coefficient of the duplex for $B_{11}$ equals that of the nulliplex for $B_{12}$, the duplex for $B_{22}$ and the quadruplex for $B_{21}$. Similar equivalents allow the changes due to double reduction in all 
TABLE 3

Genotypic proportions of generation means at limiting values of $\alpha$

\begin{tabular}{|c|c|c|c|c|c|}
\hline Generation & Quadruplex & Triplex & Duplex & Simplex & Nulliplex \\
\hline \multirow[t]{2}{*}{$P_{1}$} & 1.000 & - & - & 一 & - \\
\hline & 1.000 & 一 & - & - & - \\
\hline \multirow[t]{2}{*}{$\mathrm{P}_{2}$} & - & - & - & 一 & 1.000 \\
\hline & - & - & - & - & 1.000 \\
\hline \multirow[t]{2}{*}{$F_{1}$} & - & - & 1.000 & - & - \\
\hline & - & - & 1.000 & - & - \\
\hline \multirow[t]{2}{*}{$F_{2}$} & 0.028 & $0 \cdot 222$ & 0.500 & 0.222 & 0.028 \\
\hline & 0.046 & 0.245 & 0.420 & 0.245 & 0.046 \\
\hline \multirow[t]{2}{*}{$F_{3}$} & 0.097 & 0.222 & 0.361 & 0.222 & 0.097 \\
\hline & $0 \cdot 135$ & 0.223 & 0.285 & $0 \cdot 223$ & $0 \cdot 135$ \\
\hline \multirow[t]{2}{*}{$F_{4}$} & 0.163 & 0.191 & 0.292 & 0.191 & 0.163 \\
\hline & 0.212 & 0.179 & 0.219 & 0.179 & 0.212 \\
\hline \multirow[t]{2}{*}{$F_{2}$ bip } & 0.049 & 0.247 & 0.407 & 0.247 & 0.049 \\
\hline & 0.075 & 0.248 & 0.354 & 0.248 & 0.075 \\
\hline \multirow[t]{2}{*}{$\mathrm{F}_{2} \times \mathrm{P}_{1}$} & $0 \cdot 222$ & 0.556 & 0.222 & - & - \\
\hline & 0.274 & 0.451 & 0.274 & - & - \\
\hline \multirow[t]{2}{*}{$\mathrm{F}_{2} \times \mathrm{P}_{2}$} & - & - & 0.222 & 0.556 & 0.222 \\
\hline & - & - & 0.274 & 0.451 & 0.274 \\
\hline \multirow[t]{2}{*}{$F_{2} \times F_{1}$} & 0.037 & 0.241 & 0.444 & 0.241 & 0.037 \\
\hline & 0.059 & 0.254 & 0.376 & 0.254 & 0.059 \\
\hline \multirow[t]{2}{*}{$\mathrm{B}_{1} \times \mathrm{F}_{1}$} & 0.088 & 0.426 & 0.389 & 0.093 & 0.005 \\
\hline & 0.121 & 0.403 & 0.346 & $0 \cdot 116$ & 0.014 \\
\hline \multirow[t]{2}{*}{$\mathrm{B}_{2} \times \mathrm{F}_{1}$} & 0.005 & 0.093 & 0.389 & 0.426 & 0.088 \\
\hline & 0.014 & 0.116 & 0.346 & 0.403 & 0.121 \\
\hline \multirow[t]{2}{*}{$\mathrm{B}_{1}$} & 0.167 & 0.667 & $0 \cdot 167$ & - & - \\
\hline & 0.213 & 0.573 & 0.213 & - & - \\
\hline \multirow[t]{2}{*}{$\mathrm{B}_{2}$} & - & - & 0.167 & 0.667 & 0.167 \\
\hline & - & - & 0.213 & 0.573 & $0 \cdot 213$ \\
\hline \multirow[t]{2}{*}{$B_{11}$} & 0.528 & 0.444 & 0.028 & - & - \\
\hline & 0.566 & 0.369 & 0.066 & - & - \\
\hline \multirow[t]{2}{*}{$\mathrm{B}_{12}$} & - & - & 0.528 & 0.444 & 0.028 \\
\hline & - & - & 0.566 & 0.369 & 0.066 \\
\hline \multirow[t]{2}{*}{$\mathrm{B}_{22}$} & - & - & 0.028 & 0.444 & 0.528 \\
\hline & - & - & 0.066 & 0.369 & 0.566 \\
\hline \multirow[t]{2}{*}{$B_{21}$} & 0.028 & 0.444 & 0.528 & - & - \\
\hline & 0.066 & 0.369 & 0.566 & - & - \\
\hline \multirow[t]{2}{*}{$B_{1}$ self } & 0.338 & 0.370 & 0.250 & 0.037 & 0.005 \\
\hline & 0.387 & 0.316 & 0.217 & 0.069 & 0.010 \\
\hline \multirow[t]{2}{*}{$B_{2}$ self } & 0.005 & 0.037 & 0.250 & 0.370 & 0.338 \\
\hline & 0.010 & 0.069 & 0.217 & 0.316 & 0.387 \\
\hline \multirow[t]{2}{*}{$\mathrm{B}_{\mathbf{z}}$ bip } & 0.279 & 0.469 & 0.227 & 0.025 & 0.001 \\
\hline & 0.320 & 0.417 & 0.210 & 0.048 & 0.004 \\
\hline \multirow[t]{2}{*}{$\mathrm{B}_{2}$ bip } & 0.001 & 0.025 & 0.227 & 0.469 & 0.279 \\
\hline & 0.004 & 0.048 & 0.210 & 0.417 & 0.320 \\
\hline
\end{tabular}

Upper line $\alpha=0.0$; lower line $\alpha=0.14$. 

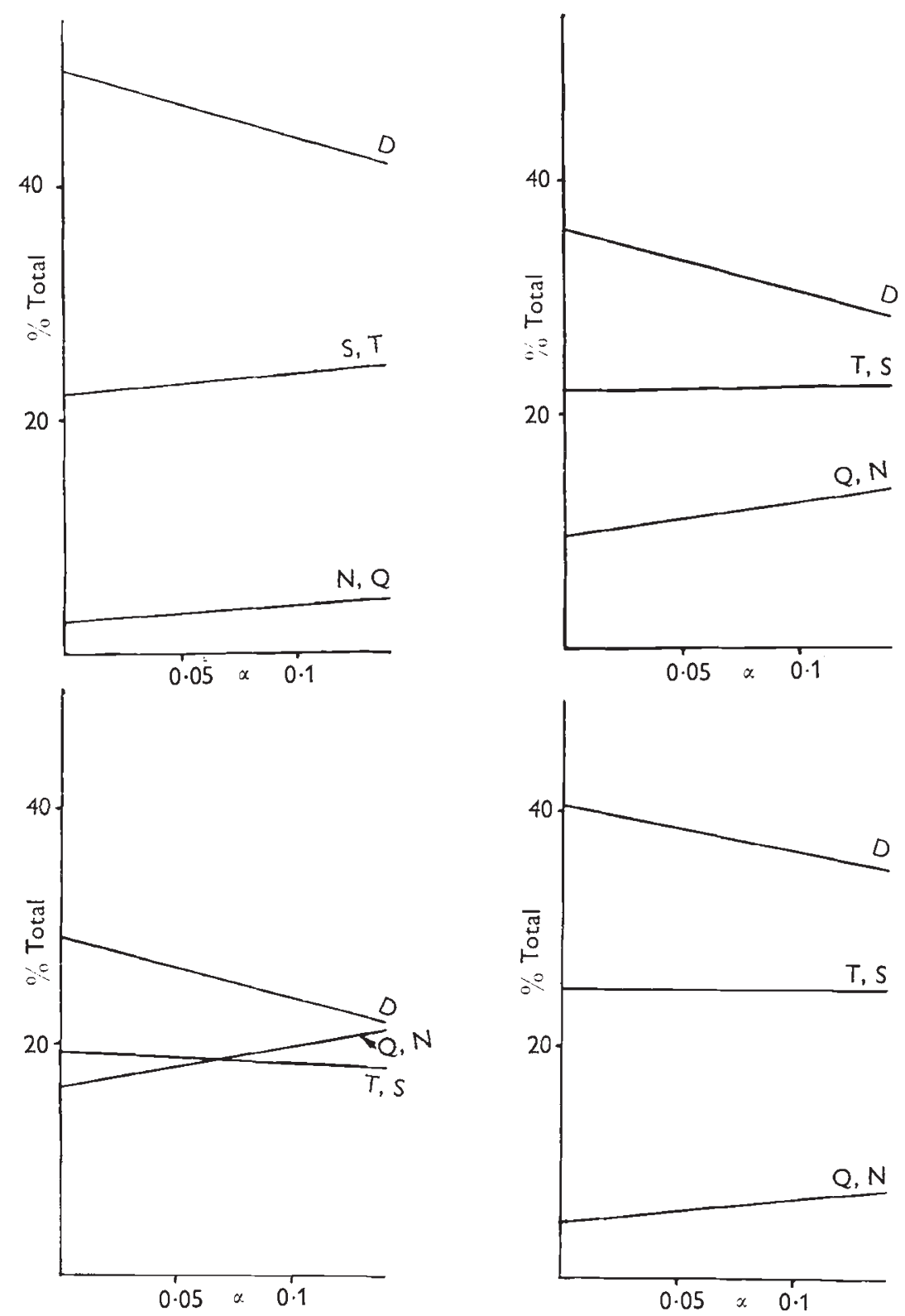

FIG 1.-Changes in the proportions of the quadruplex (Q), triplex (T), duplex (D), simplex $(\mathrm{S})$ and nulliplex $(\mathrm{N})$ genotypes with double reduction in the following generations: $\mathrm{F}_{2}$ (top left), $\mathrm{F}_{3}$ (top right), $\mathrm{F}_{4}$ (bottom left) and $\mathrm{F}_{2}$ bip (bottom right).

22 generations to be traced from figs. 1-3. The changes in frequencies of the genotypes with double reduction are linear or nearly so (where the regression is curvilinear this is so slight that it does not appear in figs. 1-3), despite the complex terms involved. This is probably due to cancellation of terms with opposite sign and to the very small range of values that $\alpha$ may 

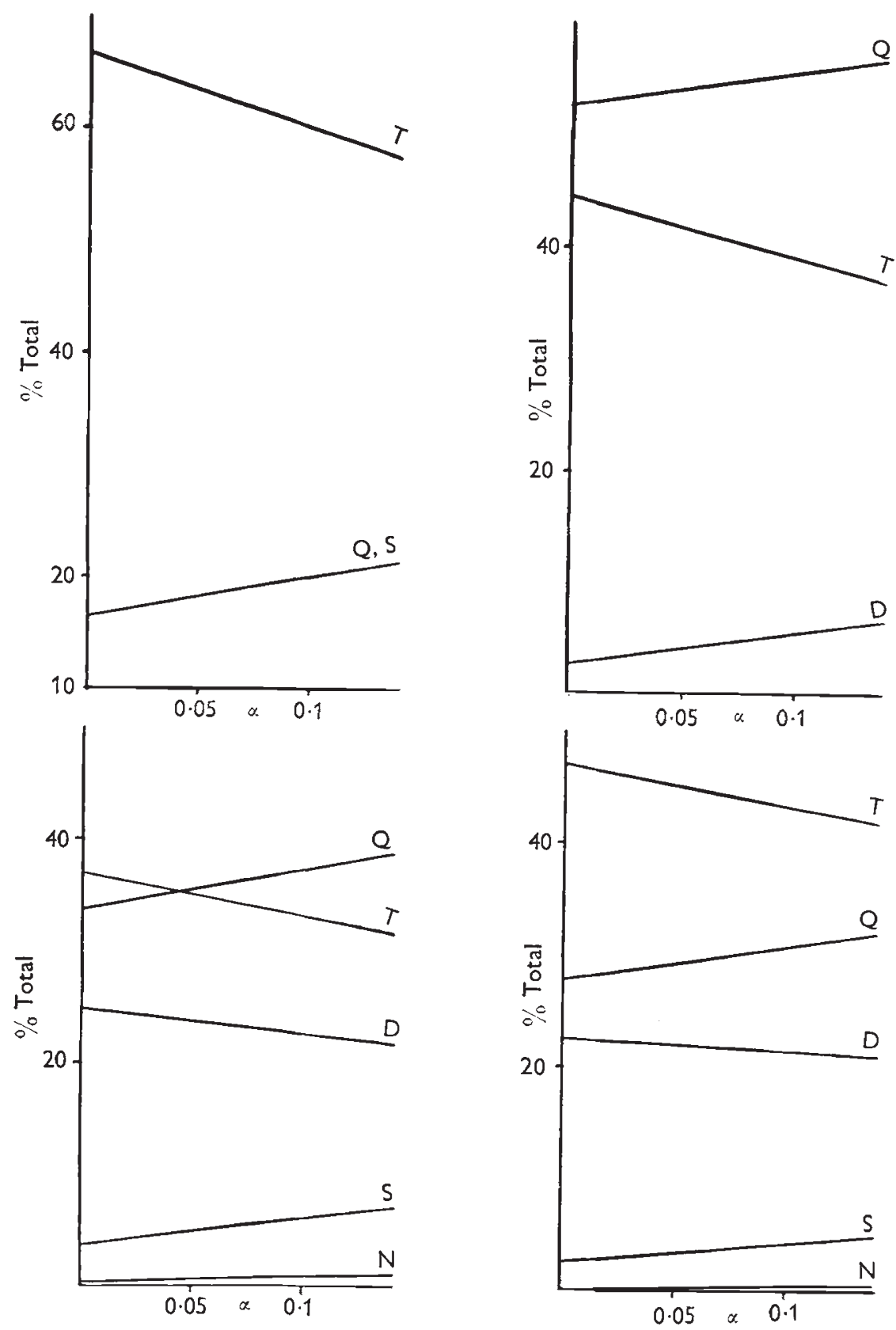

FIG. 2.-Changes in the proportions of the quadruplex (Q), triplex (T), duplex (D), simplex $(\mathrm{S})$ and nulliplex $(\mathrm{N})$ genotypes with double reduction in the following generations: $B_{1}$ (top left), $B_{11}$ (top right), $B_{1}$ selfed (bottom left) and $B_{1}$ bip (bottom right).

take; curvature would be more apparent if $\alpha$ could take values up to-sayunity. As it is, terms with high powers of $\alpha$ make a negligible contribution to the total; for instance when $\alpha=0 \cdot 14, \alpha^{3}=0 \cdot 002744$, so that terms above $\alpha^{2}$ will have little effect on the regression lines. 

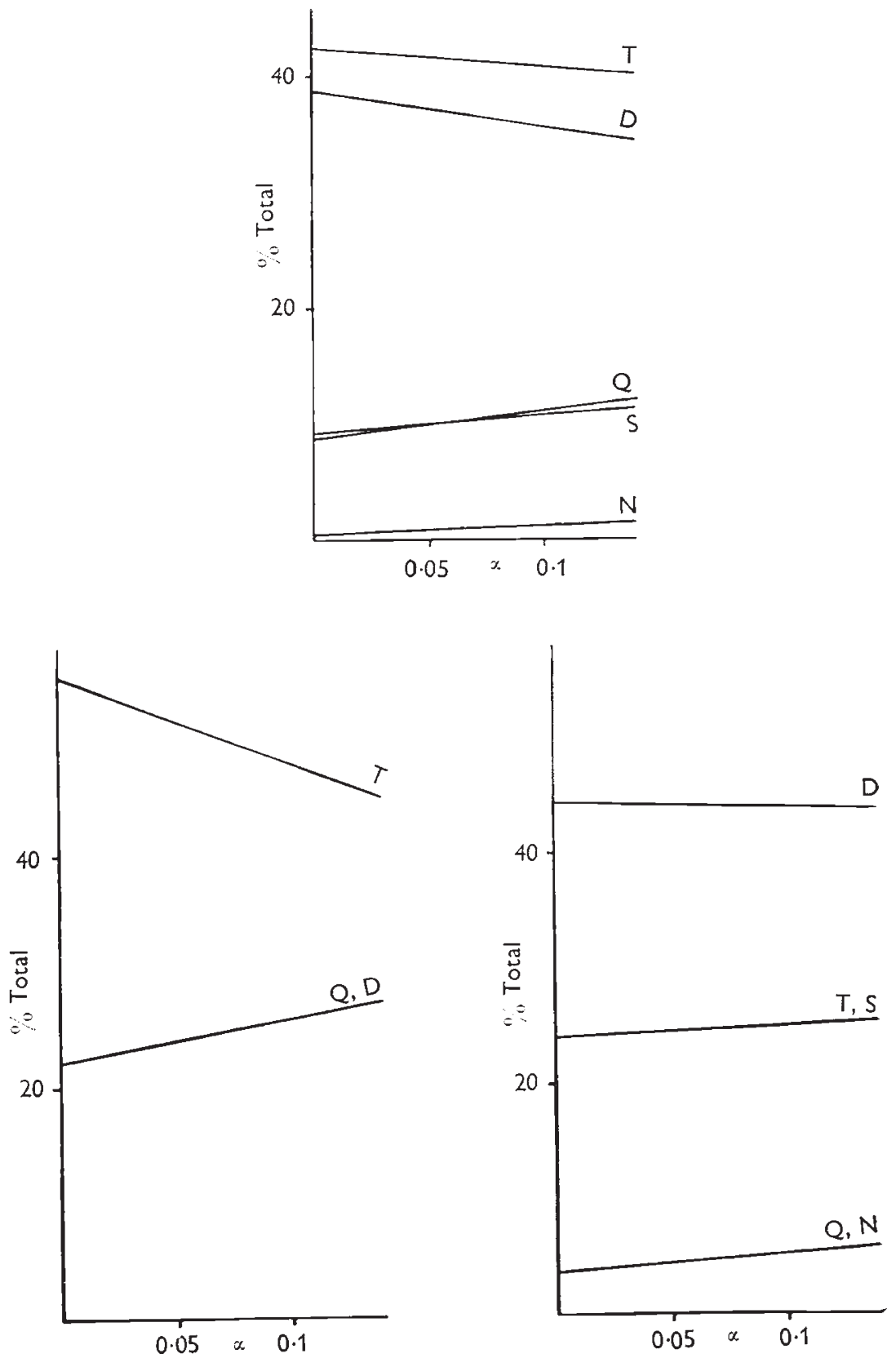

FIG. 3.-Changes in the proportions of the quadruplex (Q), triplex (T), duplex (D), simplex (S) and nulliplex (N) genotypes with double reduction in the following generations: $\mathrm{B}_{1} \times \mathrm{F}_{1}$ (top), $\mathrm{P}_{1} \times \mathrm{F}_{2}$ (bottom left) and $\mathrm{F}_{1} \times \mathrm{F}_{2}$ (bottom right).

In five generations double reduction causes the relative frequencies of genotypes to change. In the $\mathrm{F}_{4}$ the relative frequencies of quadruplex and triplex change at $\alpha=0.07$. In $\mathrm{B}_{1}$ selfed the relative frequencies of quadruplex and triplex change at $\alpha=0.04$ (in $B_{2}$ selfed it is the nulliplex and simplex 
which change). In $B_{1} \times F_{1}$ the relative frequencies of quadruplex and simplex change at $\alpha=0.08$ (in $\mathrm{B}_{2} \times \mathrm{F}_{\mathbf{1}}$ it is the nulliplex and triplex which change). The $F_{1} \times F_{2}$ generation shows little change in the proportions of the genotypes with double reduction and the triplex and simplex genotypes alter very little in the $\mathrm{F}_{3}$ and $\mathrm{F}_{2}$ bip generations. With these exceptions double reduction causes other changes in the proportions of the genotypes which would have a marked effect on the estimation of parameters from generation means.

In every generation double reduction causes the proportions of quadruplex and/or nulliplex genotypes to increase, and consequently the overall proportion of heterozygous genotypes (simplex, duplex and triplex) to decline. This is as expected since double reduction must cause inbreeding by allowing sister chromatids to enter the same gamete. Table 3 shows the actual increase in homozygosity over the whole range of $\alpha$ values. Thus in the $F_{2}$ the increase in homozygosity when $\alpha$ changes from 0.0 to 0.14 is 3.5 per cent., in the $F_{3}$ it is 7.5 per cent. and in the $F_{4}$ it is 9.8 per cent., with proportional decreases in the amount of heterozygosity. Put another way, on selfing the $F_{2}$ there is an increase in homozygosity of 13.9 per cent. if $\alpha=0.0$ and of 17.9 per cent. if $\alpha=0 \cdot 14$. On selfing the $F_{3}$ homozygosity increases by 13.1 per cent. if $\alpha=0.0$ and 15.4 per cent. if $\alpha=0.14$. Similar increases in homozygosity occur in other generations.

A secondary effect of double reduction may also be noted. With the exception of the first back-crosses selfed and the second back-crosses, double reduction increases the proportion of the rarest genotype, and decreases the proportion of the commonest genotype, in any generation. The effect is to even up the frequencies of the genotypes in the various generations, and is most marked in the selfing series, $\mathrm{F}_{2}$ bip and the first back-crosses.

\section{(c) Scaling tests}

The principle of scaling tests (Mather, 1949) is to detect deviations from additivity of gene action by combining generation means in such a way that the expected total is zero if the character is controlled only by additive and dominance gene action. Non-additivity is usually acribed to epistasis, though other genetic causes (e.g. residual heterozygosity) could be responsible for failure of the scaling tests.

Apart from the A, B and C scaling tests of Mather (1949), and the various associated tests based on the formula $\overline{\mathrm{F}}_{n}=\frac{1}{4}\left(\overline{\mathrm{P}}_{1}+\overline{\mathrm{P}}_{2}\right)+\frac{1}{2} \overline{\mathrm{F}}_{n-1}$, scaling tests have been developed by Opsahl (1956), van der Veen (1959) and Hill (1966). Using the general notation described in section 2 of this paper, none of these scaling tests is satisfactory; with only additive and dominance gene action the expected totals are not zero. Nor will any of these tests work if the three heterozygous genotypes are pooled, i.e. $h_{1}+h_{2}+h_{3}=h$.

Using the special definitions that $A_{3} a=m+\frac{1}{2} d+\frac{1}{2} h$ and $A a_{3}=m-\frac{1}{2} d$ $+\frac{1}{2} h$, six tests are satisfactory even with double reduction. These tests are

$$
\begin{aligned}
\mathrm{A} & =\overline{\mathrm{P}}_{1}+\overline{\mathrm{F}}_{1}-2 \overline{\mathrm{B}}_{1} \\
\mathrm{~B} & =\overline{\mathrm{P}}_{2}+\overline{\mathrm{F}}_{1}-2 \overline{\mathrm{B}}_{2} \quad \text { of Mather }(1949) \\
\mathrm{B}_{11} & =3 \overline{\mathrm{P}}_{1}+\overline{\mathrm{F}}_{1}-4 \overline{\mathrm{B}}_{11} \\
\mathrm{~B}_{12} & =\overline{\mathrm{P}}_{2}+3 \overline{\mathrm{F}}_{1}-4 \overline{\mathrm{B}}_{12} \\
\mathrm{~B}_{22} & =3 \overline{\mathrm{P}}_{2}+\overline{\mathrm{F}}_{1}-4 \overline{\mathrm{B}}_{22} \\
\mathrm{~B}_{21} & =\overline{\mathrm{P}}_{1}+3 \overline{\mathrm{F}}_{1}-4 \overline{\mathrm{B}}_{21} \quad \text { of Hill (1966) }
\end{aligned}
$$


Using the special definition of Dessureaux (1959), three of these tests-the $A, B_{11}$ and $B_{12}$-are satisfactory even with double reduction. Nevertheless, the reliability of these scaling tests depends on the definition of the parameters being satisfactory, which is open to doubt.

It has been possible to devise two scaling tests which are satisfactory using the general definition. These tests (which also hold for disomic inheritance) are

$$
\begin{aligned}
& \overline{\mathrm{P}}_{1}-\overline{\mathrm{F}}_{1}+2 \overline{\mathrm{B}}_{21}-2 \overline{\mathrm{B}}_{11}=0 \\
& \overline{\mathrm{P}}_{2}-\overline{\mathrm{F}}_{1}+2 \overline{\mathrm{B}}_{12}-2 \overline{\mathrm{B}}_{22}=0 .
\end{aligned}
$$

The variances of these tests are

$$
\begin{aligned}
& \mathrm{V}_{\overline{\mathrm{P}}_{1}}+\mathrm{V}_{\overline{\mathrm{F}_{1}}}+4 \mathrm{~V}_{\overline{\mathrm{B}_{21}}}+4 \mathrm{~V}_{\overline{\mathrm{B}_{11}}} \text { and } \\
& \mathrm{V}_{\overline{\mathrm{P}}_{2}}+\mathrm{V}_{\overline{\mathrm{F}}_{1}}+4 \mathrm{~V}_{\overline{\mathrm{B}_{12}}}+4 \mathrm{~V}_{\overline{\mathrm{B}_{22}}} \text { respectively. }
\end{aligned}
$$

These two scaling tests are unaffected by the degree of double reduction The scaling test of Cavalli (1952) can, of course, be adapted to any generation and any type of inheritance. It would therefore be quite satisfactory when applied to tetraploid data.

\section{Second-Degree statistics}

Using the notation that $d=\frac{1}{2}\left(A_{4}-a_{4}\right), h_{1}=A a_{3}, h_{2}=A_{2} a_{2}$ and $h_{3}$ $=A_{3} a$, the expectations of second-degree statistics may be calculated. Thus the $\mathrm{F}_{2}$ variance is

$$
\begin{aligned}
& \frac{1}{18}\left(1+4 \alpha+4 \alpha^{2}\right) d^{2}+\frac{1}{81}\left(14+10 \alpha-24 \alpha^{2}+16 \alpha^{3}-16 \alpha^{4}\right)\left(h_{1}^{2}+h_{3}^{2}\right)+\frac{1}{36} \\
& \left(9-16 \alpha^{2}+32 \alpha^{3}-16 \alpha^{4}\right) h_{2}^{2}+\frac{1}{27}\left(-6+2 \alpha+12 \alpha^{2}-24 \alpha^{3}-16 \alpha^{4}\right)\left(h_{1} h_{2}\right. \\
& \left.+h_{2} h_{3}\right)+\frac{1}{81}\left(-8-16 \alpha+24 \alpha^{2}+32 \alpha^{3}-32 \alpha^{4}\right) h_{1} h_{3} .
\end{aligned}
$$

Similarly, the variance of $B_{1}$ is

$$
\frac{1}{36}\left(5+8 \alpha-4 \alpha^{2}\right)\left(d^{2}+h_{2}^{2}\right)+\frac{1}{9}\left(2+2 \alpha-4 \alpha^{2}\right)\left(h_{3}^{2}-h_{2} h_{3}-d h_{3}\right)-\frac{1}{18}\left(1-4 \alpha+4 \alpha^{2}\right) d h_{2} .
$$

Whilst all second-degree statistics could be calculated taking double reduction into account there would appear little to be gained for the extra labour required. The results given here have therefore been based on the special case where $\alpha=0.0$ (i.e. segregation is chromosomal). Many statistics can be derived from the 22 generations listed earlier in this paper, but attention here will be restricted to statistics obtainable from the selfing series, $\mathrm{F}_{2}$ bip, the first and second back-crosses and the first back-crosses selfed. The genetical expectations of these statistics are given in table 4 . Statistics such as the covariance of $\mathrm{B}_{12}$ and $\mathrm{B}_{1}$ selfed can, of course, only be obtained by using common parents; where fertility is low this may not be possible. Ten parameters need to be estimated though if all the $h$ and $d h$ terms are summed this can be reduced to three, in which case the assumption that all the terms in $h$ are equal $\left(h_{1}=h_{2}=h_{3}\right)$ has been made, and a possible error introduced.

Apart from the $F_{2}$ the coefficients of the parameters in the selfing series are very complex and involve terms in $d h$, though since the coefficients of $d h$ are of the same size but opposite sign these disappear from the model on summation of the parameters. However if $h_{3}$ is not equal in effect to $h_{1}$, 


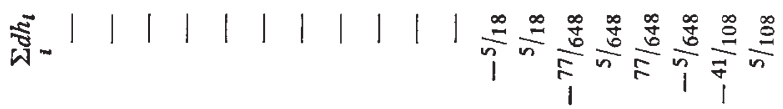

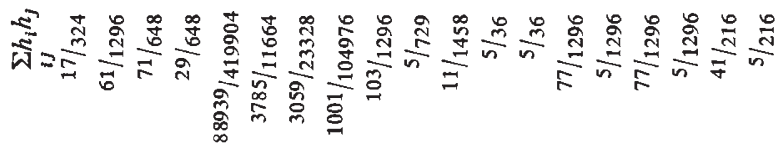

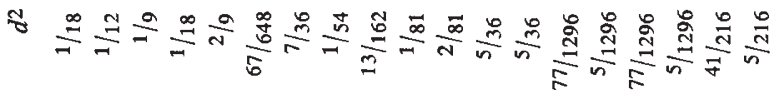

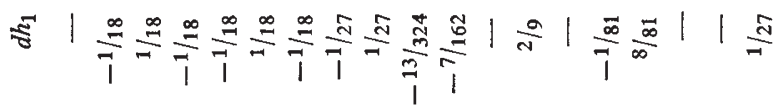
密 旁

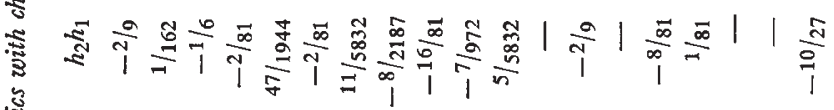

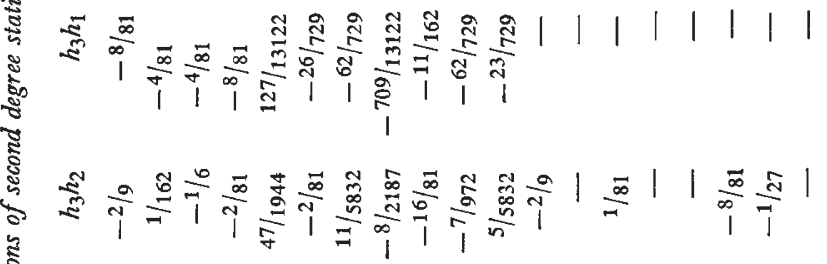

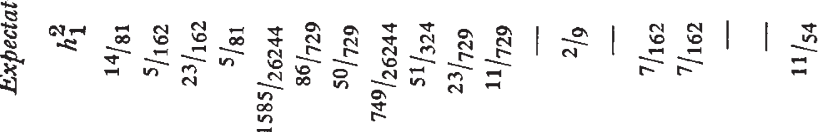

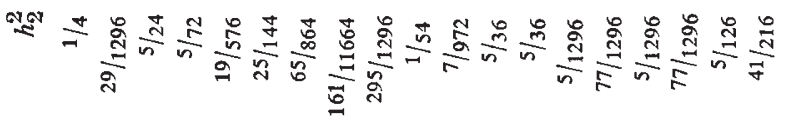

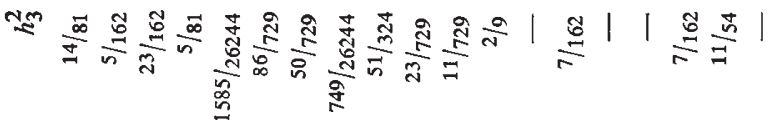

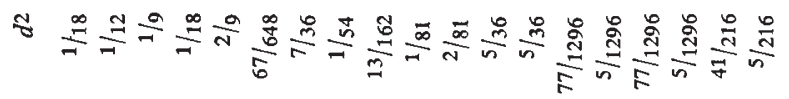

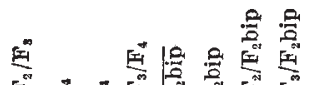

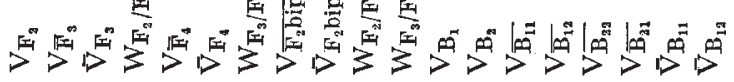




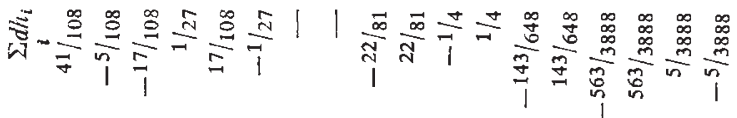

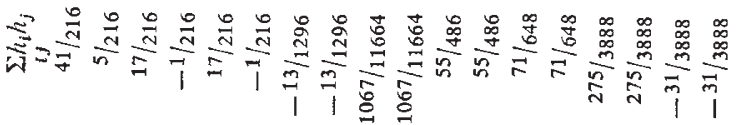

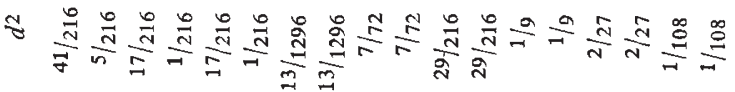

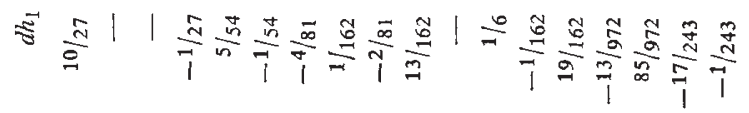

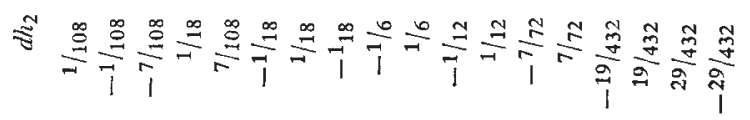

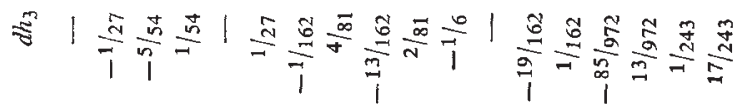

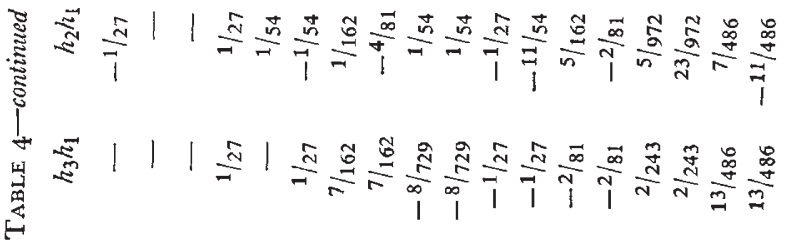
跑1

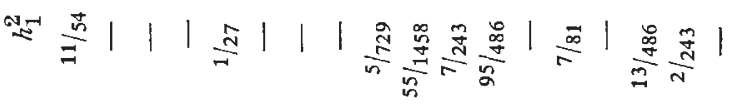

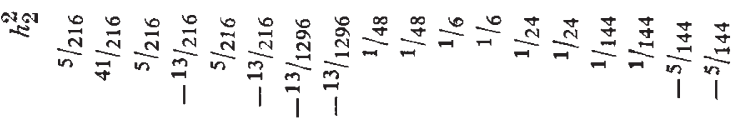

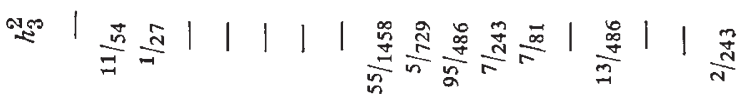

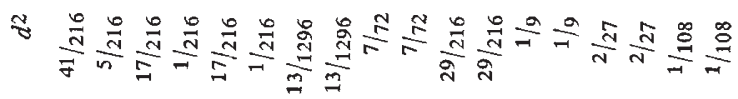

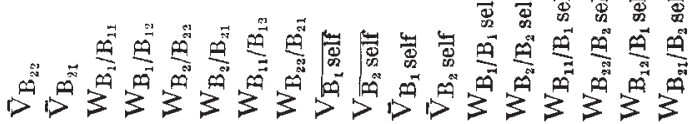


genic effects of $d h$ terms will still exist in the data though they are not allowed for in the model. The sign of $d h$ is positive for the mean variances and negative for the variance of means and covariance statistics. As with the mean of the $\mathrm{F}_{2}$ and $\mathrm{F}_{2}$ bip the variance of the $\mathrm{F}_{2}$ does not equal the total $\mathrm{F}_{2}$ bip variance $\left(\overline{\mathrm{V}} \mathrm{F}_{2}\right.$ bip $+\mathrm{VF}_{2}$ bip $)$. This is expected since in polyploids a single round of random mating is not sufficient to bring a population into equilibrium (Bennet, 1954).

The first and second back-cross statistics do not use all ten parameters simultaneously, so that separation of their effects should be easier. It may be noted that here, as in diploids, the coefficient of $d^{2}$ is equal to that of $\sum_{i j} h_{i} h_{j}$ so that additive and dominance effects may be estimated with equal precision.

The variances may be estimated by either of two procedures.

(a) By using the analysis of variance the phenotypic variation of each generation (except the $\mathrm{F}_{2}$ and first back-crosses) may be partitioned into that within families and that between families. The two mean squares have expectations of:

$$
\begin{aligned}
& \text { Between families M.S. }=\sigma_{w}^{2}+n \sigma_{b}^{2} \\
& \text { Within families M.S. }=\sigma_{w}^{2}
\end{aligned}
$$

where $n$ is the number of individuals per family. The two $\sigma^{2}$ 's may thus be separated and ascribed the genetical expectations shown in table 4. Assuming single plant randomisation, the expectation of the within family variance, $\sigma_{w}^{2}$, also contains an environmental component, $\mathrm{E}_{1}$, which is estimated as the mean variance within parental and $\mathrm{F}_{1}$ lines.

(b) By direct estimation of the phenotypic variances within and between families for each generation. For the latter statistic the data used are the family means so its expectation is $\frac{1}{n}$ of the between families mean square in the analysis of variance $\left(i . e . \frac{1}{n} \sigma_{w}^{2}+\sigma_{b}^{2}\right.$ ). Whereas the within family variance is identical when estimated by either procedure, the expectation of the between families variance estimated by method $(b)$ is incremented by $\frac{1}{n}$ of the within families variance and this includes $\mathrm{E}_{\mathbf{1}}$. Therefore when method $(b)$ is used to estimate the between families variance of any generation a correction needs to be made before the genetical expectation in table 4 is ascribed. If individual plant randomisation is not used, the expectations of thevariances between families also contain an environmental component, $\mathrm{E}_{2}$, but this is zero with single plant randomisation.

The main difficulty in estimating parameters from the variances is likely to be the small, but variable, coefficients involved. If we consider as an example the variance of second back-cross family means we see that the coefficients of $d^{2}, \sum_{i j} h_{i} h_{j}$ and $\sum_{i} d h_{i}$ are some 15 times greater for $\mathrm{B}_{11}$ and $\mathrm{B}_{22}$ than for $\mathrm{B}_{12}$ and $\mathrm{B}_{21}$. In the case of the mean variances of second backcrosses the coefficients of the summed parameters $\left(d^{2}, \sum_{i j} h_{i} h_{j}, \sum_{i} d h_{i}\right)$ of $B_{11}$ and $B_{22}$ are about eight times those of $B_{12}$ and $B_{21}$. So that for $\bar{\nabla}_{B_{11}}$ and 
$\bar{V}_{\mathrm{B}_{22}}$ the coefficients of the genetical parameters are about a fifth (41/216) that of $E_{1}$ and for $\bar{V}_{B_{12}}$ and $\bar{V}_{B_{21}}$ they are about a fortieth (5/216) that of $\mathrm{E}_{1}$. There is clearly a possibility that, in practice, the genetical variation will be "drowned" by the environmental variation and that the estimates of the genetical parameters will be imprecise. This does not apply to covariances which have no environmental component in their expectations (Mather, 1949).

\section{Discussion}

Scaling tests provide unambiguous qualitative information as to the presence or absence of epistasis (Perkins and Jinks, 1970). It is therefore possible to assess the adequacy of a simple additive and dominance model before estimating any parameters. If the scaling tests described in this paper show epistasis to be absent, the components of generation means may be estimated using the method of Cavalli (1952). The components are $m$, the midparent value, $d, h_{1}, h_{2}$ and $h_{3}$ and their products with $\alpha$ to various powers. Excluding the $\mathrm{F}_{4}$ mean there are potentially 21 such parameters, and if the $\mathrm{F}_{4}$ mean is included there are 27 parameters (because here we need to specify $\alpha^{5} h_{1}, \alpha^{6} h_{1}$, etc.). Since we have only 22 generation means from which to estimate these parameters the $\mathrm{F}_{4}$ must obviously be excluded. The model to be fitted can be varied in complexity until a satisfactory fit is obtained. Thus at first the model might only contain the parameters $m, d, h_{1}, h_{2}$ and $h_{3}$. If the fit is unsatisfactory (i.e. the $\chi^{2}$ is too large) the parameters $\alpha d, \alpha h_{1}, \alpha h_{2}$ and $\alpha h_{3}$ may be added. Parameters with successively higher powers of $\alpha$ may continue to be added until a satisfactory fit is obtained. A description of this approach applied to diploids is given by Jinks and Perkins (1969) for the estimation of linked epistatic parameters from generations means. The linearity of the regression lines in figs. 1-3 suggest that it would be unnecessary to fit parameters with $\alpha$ raised to a power greater than l. Nine parameters would therefore have to be fitted and so ten (or more) generation means may be adequate for the purpose of estimating them. Comparison of the relative sizes of $h_{1}, h_{2}$ and $h_{3}$ may indicate that one of the special definitions of these parameters described in section 2 of this paper may be appropriate in which case a simpler model may be fitted.

Ten parameters are specified in the expectations of second-degree statistics, and hence at least ten statistics are required for their estimation. These parameters have been shown to have relatively small coefficients and hence their estimation will be subject to a large error. If the analysis of generation means suggests that $h_{1}, h_{2}$ and $h_{3}$ can be combined into a single parameter the number of second-degree parameters is reduced from ten to three and the coefficients are larger, though still small in comparison with that of the environmental component. Consequently the analysis will be easier and more accurate if such simplification is justified. Since covariances do not have an environmental component in their expectations they are potentially more useful than variances. A common drawback to the use of covariances is that they frequently involve the use of two non-contemporary generations, e.g. W $\mathrm{F}_{2} / \mathrm{F}_{3}$, and therefore the measurements must be made in different seasons causing the statistics to be distorted by genotype-environment interactions. Fortunately several polyploids (e.g. potatoes) can be propagated vegetatively so the parent may be measured at the same time, and under the 
same conditions, as its progeny. Even so, it would seem that in tetraploids first-degree statistics are likely to be a more fruitful source of information than second-degree statistics.

Polyploids are frequently found to be highly heterozygous and difficulty may be experienced in obtaining homozygous lines. Selfing an autotetraploid to-obtain inbred lines is slower than selfing a diploid (see table 3); to attain the same degree of homozygosity as a diploid takes 3.8 times as long with no double reduction and 2.9 times as long if $\alpha=0.14$ (Parsons, 1959). Seven or eight generations of selfing are required to make an initially heterozygous diploid 99 per cent. homozygous (Fisher, 1949). Parsons (1959) shows that tetraploids require 27 or 28 generations of selfing to achieve the same degree of homozygosity if $\alpha=0$; if $\alpha=0 \cdot 14,20$ generations of selfing will suffice. It would clearly be worth while to reduce the tetraploid to the diploid level, inbreed for several generations and then double the number of chromosomes using colchicine, provided it was technically feasible. However, other difficulties may occur. For instance, diploid potatoes have a selfincompatibility system which is not expressed in the tetraploid forms. The most rigorous form of inbreeding which could be applied to such diploids is sib-mating, which is 3.3 times slower than selfing a diploid in the rate of approach to homozygosity. Sib-mating a diploid would be faster than selfing the tetraploid only if $\alpha<0.07$ (Parsons, 1959), so that no real advantage would acrue from attempting to inbreed at the diploid level if a self-incompatibility system operated.

\section{Summary}

1. The suitability of several models for the study of quantitative inheritance in autotetraploids is considered.

2 . The expectations of generation means are given in terms of the proportions of quadruplex, triplex, duplex, simplex and nulliplex genotypes in each, and the effect double reduction has on these proportions is described.

3. A scaling test for detecting epistasis in autotetraploids is given.

4. The expectations of several second-degree statistics is given and problems of estimating parameters from them is discussed.

Acknowledgments. - I am grateful to Mr J. L. Fyfe for his helpful comments and to Miss M. A. Turner for preparing the diagrams in this paper.

\section{REFERENCES}

BennetT, J. H. 1954. Panmixia with tetrasomic and hexasomic inheritance. Genetics, 39, 150-158.

CAVALL1, L. L. 1952. An analysis of linkage in quantitative inheritance. In Quantitative Inheritance, pp. 135-144. H.M.S.O.

Dessureaux, L. 1959. Introduction to the autotetraploid diallel. Can. 7. Genet and Cyt., 1, 94-101.

FISHER, R. A. 1949. The Theory of Inbreeding. Oliver and Boyd, Edinburgh.

HILL, J. 1966. Recurrent backcrossing in the study of quantitative inheritance. Heredity, 21, 85-120.

JINKS, J. L., AND PERKINS, J. M. 1969. The detection of linked, epistatic genes for a metrical trait. Heredity, 24, 465-475.

MATHER, K. 1936. Segregation and linkage in autotetraploids. F. Genet., 32, 287-314.

MATHER, K. 1949. Biometrical Genetics. Methuen, London. 
MATHER, K., AND JINKS, J. L. 1971. Biometrical Genetics. Second edition. Chapman Hail, London.

OPSAHL, B. 1956. The discrimination of linkage and interaction in continuous variation. Biometrics, 12, 415-432.

PARSONS, P. A. 1959. Some problems in inbreeding and random mating in tetrasomics. Agron. F., 51, 465-467.

PERKINS, J. M., AND JINKS, J. L. 1970. The detection and estimation of genotype environment interaction, linkage and epistatic components of variation for a metrical trait. Heredity, $25,157-177$.

VAN DER VEEN, J. H. 1959. Tests of non-allelic interactions and linkage for quantitative characters in generations derived from two diploid pure lines. Genetica, 30, 201-232. 